is an issue). Every learner completes a knowledge, skills and confidence audit and the results are outstanding, an average increase of $60 \%$ in knowledge, skills and confidence for all learning objectives on every session. Each module is supported by two visits, one for implementation ('show me') one for evidence of the implementation of learning in practice ('prove it').

By using real experiences, the sessions are relevant, true and goals are realistic, often using the mantra ' $I$ can do it so can you', staff soon learn that behind every 'Fred' and 'Doris' (safe names used on each session for confidentiality) is a real person; their legacy is their story, a gift for your learning.

Staff in all care homes I am supporting with the programme have evidence of residents' stories, the impact on all those important to them is enormous.

To conclude, end of life care education for care home staff need not be dull, text book or irrelevant. We need our care home staff to feel motivated and empowered, that is exactly the aim of Six Steps Too...

\section{P-175 HERTS NEIGHBOURS VOLUNTEER NURSING HOME SUPPORT}

Liz Kennedy. Peace Hospice Care, Watford, UK

\subsection{6/bmjspcare-2019-HUKNC.197}

Background Loneliness and social isolation are different but related concepts. Social isolation can lead to loneliness and loneliness can lead to social isolation. They share many factors associated with increasing the likelihood of people experiencing deteriorating health, sensory and mobility impairments (Age UK, 2018).

Following embedding Herts Neighbours volunteer befriending home support, it was identified patients whose permanent place of residence was a nursing home $(\mathrm{NH})$ were not accessing the service.

Aims Uphold our strategic aims and values 'widening access', 'reaching more people'; 'each person gets fair access to care'(Ambitions for palliative and end of life care, National Palliative and End of Life Care Partnership, 2015). Pilot agreed to extend existing model into local nursing home.

Methods February 2018 - Consultation: volunteers and nursing home staff to identify those participating. Volunteer agreement between both organisations. Criteria and consent for pilot inclusion agreed.

June 2018: five patients and three volunteers identified 12 visits made

- Pre- and post- feedback questionnaire;

- Regular support for volunteers from hospice and identified links at nursing home;

- Qualitative feedback from patients, family, staff.

Results Though the sample was small, improvements were identified:

- Loneliness $83 \%$ positive impact;

- Enjoyment of life $83 \%$ positive impact;

- Quality of life 50\% positive impact;

- Specific examples received from family, staff.

Conclusion Evaluation of the pilot showed a positive impact and outcome on quality of life, wellbeing and feelings of loneliness. We learnt that applying the same volunteer coordinator assessment in line with pre-existing process would strengthen the referral and patient feedback completion. Feedback identified actions to improve access and support service. Phase 2 agreed intending to strengthen and reach more people in the future.

Future planning: Consider development of project in line with the outcome of Population Based Needs Assessment report completed 2019.

Project and outcome presented to Internal Governance, Trustee and volunteer groups. Continuing with the project is recommended.

\section{P-176 AN INITIATIVE TO IMPROVE PALLIATIVE CARE PROVISION IN RURAL CARE HOMES}

1,2Suzanne Rainsford, ${ }^{1,2}$ Nicholas Glasgow, ${ }^{2}$ Nikki Johnston. 'Australian National University, Canberra, Australia; ${ }^{2}$ Calvary Health Care - Clare Holland House, Canberra, Australia

\subsection{6/bmjspcare-2019-HUKNC.198}

Background Palliative care in care homes is mostly primary care, with limited access to specialist support, especially in rural settings. To plan for, and recognise anticipated events, thus preventing potentially avoidable hospitalisation and allowing residents to die 'well', requires new approaches to upskill staff. An emerging model, not yet trialled in rural settings, is the introduction of outreach specialist palliative care needs rounds (Chapman, Johnston, Lovell, Forbat et al., 2018; Johnston, Lovell, Liu, Chapman et al., 2019).

Aim To explore, through the experiences of staff and GPs, the provision of end-of-life care within rural care homes prior to, and after the introduction of needs rounds (or case-based educational monthly triage meetings with a palliative medicine specialist [physician] and care home staff).

Design This presentation reports the qualitative inductive data from a larger ethics approved quasi-experimental mixed method study. This ethnographic component utilised 14 open-ended interviews, conducted between 1 March and 18 November 2018, and observational notes. Eleven needs rounds were conducted, commencing 6 April 2018; 25 residents under the care of nine GPs were discussed; 18 care home staff participated in one or more needs rounds. Interviews were audio-recorded, transcribed, and analysed thematically.

Participants: Eight care home staff, working in two facilities, and 3 GPs located in a rural town (population 6,700) in south-eastern NSW, Australia.

Results Participants identified system and site-specific barriers to quality end-of-life care. Needs rounds strengthened: (1) awareness of end of life, reflective practice, and critical thinking by care workers; (2) end-of-life decision making and planning; (3) end-of-life pain management.

Conclusion This study provides a rural perspective on the needs rounds model of care in care homes. While the model of care strengthened the knowledge and confidence of care home staff in providing end-of-life care, its sustainability requires collaboration, co-ordination and increased engagement with GPs. Suggestions for future collaboration through multidisciplinary educational case conferences will be presented. 